\title{
FT-IR Studies of Cu Substituted Ni-Zn Ferrites for Structural and Vibrational Investigations
}

\author{
M. KALYAN RAJU \\ Department of Physics, Andhra University, Visakhapatnam-530003, A. P., India \\ kalyanrs09@gmail.com
}

Received 27 September 2014 / Accepted 17 October 2014

\begin{abstract}
Polycrystalline ferrite samples having a chemical formula $\mathrm{Ni}_{0.7-\mathrm{x}} \mathrm{Cu}_{\mathrm{x}} \mathrm{Zn}_{0.3} \mathrm{Fe}_{2} \mathrm{O}_{4}$ (where $\mathrm{x}=0.05$ to 0.50 insteps of 0.05 variation) was prepared through the conventional ceramic method. The infra red spectra were recorded at the room temperature using FT-IR spectra for all samples in the range $4000 \mathrm{~cm}^{-1}$ to $400 \mathrm{~cm}^{-1}$ on MAGNA 550 Nicollet Instruments Corporation; the absorption spectra recorded $400 \mathrm{~cm}^{-1}$ to $4000 \mathrm{~cm}^{-1}$. The spectrum recorded transmittance (\%) versus wave number $\left(\mathrm{cm}^{-1}\right)$ and the spectrum confirms the spinel structure. The study of FT-IR spectra is very important tool for getting the ions positions in the lattice.
\end{abstract}

Keywords: FT-IR spectra, Copper substitution, Vibrational investigations

\section{Introduction}

Polycrystalline magnetic components must be miniaturized to reduce device size in communication systems. The multilayer chip inductor was developed to increase the volume efficiency. They are important components in the latest products, such as notebooks, cellular phones, etc. The advantages of these chip devices over conventional wire wound components are excellent magnetic shielding and miniaturization. This chip device can suppress high harmonic noise to the greatest extent. The infrared spectroscopic technique is based upon the fact that a chemical substance shows marked selective absorption in the infrared region. Various bands present in IR spectrum correspond to the characteristic functional groups and bonds present in the chemical substance. The infrared spectra is useful to determining the local symmetry and non crystalline solids and to study the ordering phenomena in ferrite.

\section{Experimental}

The $\mathrm{Cu}$ substituted NiCuZn ferrites were prepared by Conventional Ceramic Technique (CCT). Analytical reagent grade $\mathrm{NiO}, \mathrm{CuO}, \mathrm{ZnO}$ and $\mathrm{Fe}_{2} \mathrm{O}_{3}$ were weighed in the composition of $\mathrm{Ni}_{0.7-\mathrm{x}} \mathrm{Cu}_{\mathrm{x}} \mathrm{Zn}_{0.3} \mathrm{Fe}_{2} \mathrm{O}_{4}$. These oxides were intimately mixed through agate mortar with the medium of methanol. The ground powder calcined at $900{ }^{\circ} \mathrm{C}$ for 4 hours at air atmospheric using programmable furnace. The pre-sintered powder were mixed with $5 \mathrm{Wt} \%$ 
polyvinyl alcohol (PVA) as a binder and uniaxially pressed into toroids and pellets at pressure of a 2.5 tone/ $\mathrm{cm}^{2}$, respectively, the compacts and powders were successfully sintered in air atmosphere at $1100{ }^{\circ} \mathrm{C}$ for four hours and finally cooled to a room temperature. Finally the chemical characteristics of NiCuZn ferrites were determined using Fourier transform infrared spectroscopy.

\section{Results and Discussion}

The electrical and magnetic properties of spinels depend on the chemical composition, cation distribution and the method of preparation. The vibrational, electronic and magnetic dipole spectra can give information about the position and valency of the ions in the crystal lattice. Ferrites possess the structure of mineral spinel $\left(\mathrm{MgAl}_{2} \mathrm{O}_{4}\right)$ that crystallizes in the cubic form with space group ${ }^{1} \mathrm{Fd}_{3} \mathrm{~m}-\mathrm{O}_{\mathrm{h}} 7$. The spinel ferrites have four infrared active bands, designated as $\vartheta_{1}, \vartheta_{2}, \vartheta_{3}$ and $\vartheta_{4}$. The first three bands are observed due to tetrahedral and octahedral complexes while the fourth one is due to some type of lattice vibrations ${ }^{2}, 3$. Waldron ${ }^{4}$ attributed the band $\vartheta_{1}$ around $600 \mathrm{~cm}^{-1}$ to the intrinsic vibration of tetrahedral metal oxygen complexes and band $\vartheta_{2}$ at around $400 \mathrm{~cm}^{-1}$ to the intrinsic vibration of octahedral metal oxygen complexes. These bands are mainly depending on Fe-O distances.

It is observed that each spectrum (Figure 1) consists of two significant absorption bands which reveal the formation of single phase of spinel ferrites having two sub lattices, tetrahedral site and octahedral site 5 . The occurrence of first band at higher wave number of $\sim 583 \mathrm{~cm}^{-1}\left(\vartheta_{1}\right)$ has been assigned to the intrinsic vibrations of the tetrahedral complexes corresponding to the highest restoring force. Whereas the second band at lower wave number of $\sim 401 \mathrm{~cm}^{-1}\left(\vartheta_{2}\right)$ is attributed to the intrinsic vibrations of the octahedral complexes.

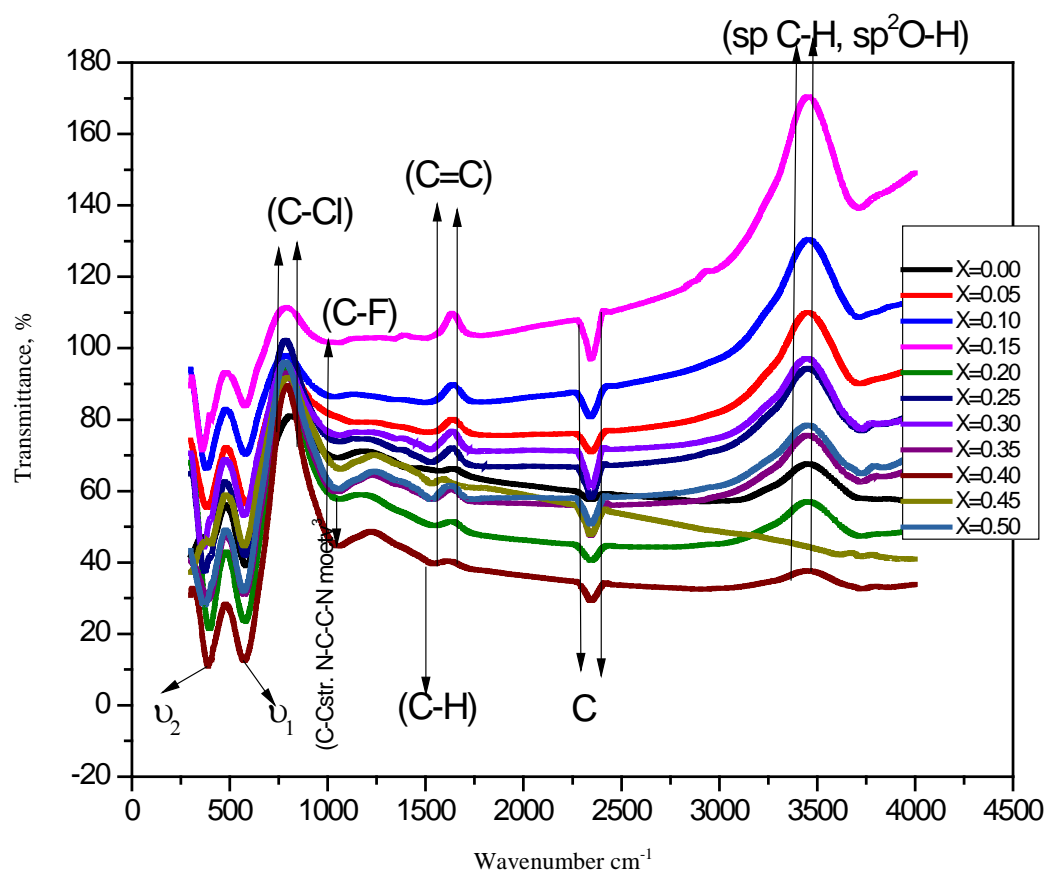

Figure 1. FTIR absorption spectra of $\mathrm{Ni}_{0.7-\mathrm{x}} \mathrm{Cu}_{\mathrm{x}} \mathrm{Zn}_{0.3} \mathrm{Fe}_{2} \mathrm{O}_{4}$ ferrites 
The difference in frequencies of the characteristic vibrations $\left(\vartheta_{1} \& \vartheta_{2}\right)$ has been attributed to the long bond length of oxygen-metal ions in the octahedral sites and shorter band length of oxygen-metal ions in the tetrahedral sites ${ }^{6}$. The bands at $3430 \mathrm{~cm}^{-1}$ and $1540 \mathrm{~cm}^{-1}$ are due to the $\mathrm{O}-\mathrm{H}$ stretching vibrations significant reduction shows that the Fe$\mathrm{O}-\mathrm{H}$ for ferrite powder and which was caused by the free absorbed water ${ }^{7}$.

Figure 2 shows the spectra between the wave numbers $300 \mathrm{~cm}^{-1}$ to $1000 \mathrm{~cm}^{-1}$ which is represents the formation of the ferrite spinel. Here two significant bands are present $\vartheta_{1} \&$ $\vartheta_{2}$. The higher wave number $\vartheta_{1}$ band is in between $550 \mathrm{~cm}^{-1}$ to $600 \mathrm{~cm}^{-1}$ and it is caused by the stretching vibrations of metal-oxygen bond which is tetrahedral (A) sites. The lower wave number range is $365 \mathrm{~cm}^{-1}$ to $425 \mathrm{~cm}^{-1}$ is caused by M-O bond vibrations in the octahedral [B] sites ${ }^{8}$. The stretching vibrations at $1633 \mathrm{~cm}^{-1}$ is corresponding water deformation $\mathrm{H}_{2} \mathrm{O}$ and it indicates the hydrogen bond ${ }^{9,10}$ and is due to the $\mathrm{O}-\mathrm{H}$ stretching vibration bond. The positions of absorption bands in terms of wave number $\left(\vartheta_{1} \& \vartheta_{2}\right)$ along with intensities of absorption bands for all the copper substituted samples are given in Table 1.

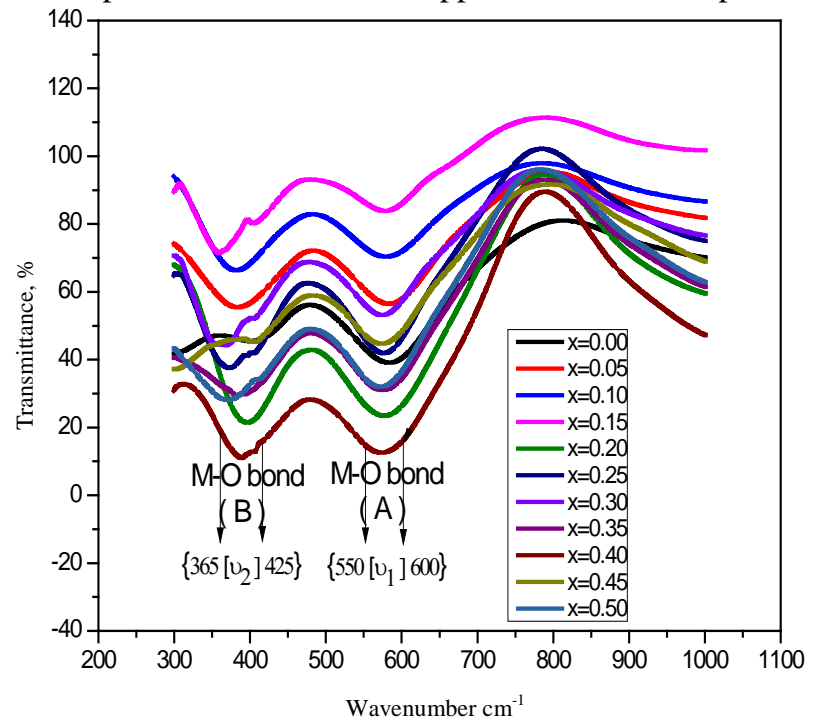

Figuire 2. IR Spectra of the ferrite spinel

Table 1. Absorption band positions and their respective intensities

\begin{tabular}{ccccc}
\hline $\mathrm{X}$ & $\vartheta_{1}, \mathrm{~cm}^{-1}$ & $\mathrm{I}_{1}, \%$ & $\vartheta_{2}, \mathrm{~cm}^{-1}$ & $\mathrm{I}_{2}, \%$ \\
\hline 0.00 & 582.5 & 39 & 401.19 & 45.39 \\
0.05 & 582.5 & 56.16 & 383.83 & 54.99 \\
0.10 & 578.64 & 69.96 & 381.9 & 65.83 \\
0.15 & 578.64 & 83.56 & 358.76 & 71.17 \\
0.20 & 576.72 & 23.19 & 395.4 & 21.39 \\
0.25 & 576.72 & 41.42 & 374.19 & 37.49 \\
0.30 & 574.79 & 52.69 & 368.4 & 44.43 \\
0.35 & 574.79 & 30.67 & 381.91 & 29.49 \\
0.40 & 574.79 & 11.28 & 389.62 & 10.65 \\
0.45 & 574.79 & 44.40 & - & - \\
0.50 & 572.86 & 31.95 & 369.11 & 28.02 \\
\hline
\end{tabular}


The variation in wave numbers $\left(\vartheta_{1} \& \vartheta_{2}\right)$ with copper concentration(x) for all samples is shown in Figure 3. From the figure, it has been observed that both $\vartheta_{1}$ and $\vartheta_{2}$ shift towards lower frequencies with copper concentration (x) due to sintering on the samples ${ }^{11}$. The values of the force constants $\left(\mathrm{K}_{\mathrm{T}}\right.$ and $\left.\mathrm{K}_{\mathrm{O}}\right)$ for the band $\mathrm{Fe}^{3+}-\mathrm{O}^{2-}$ at tetrahedral and octahedral sites are calculated using the relation ${ }^{12}$.

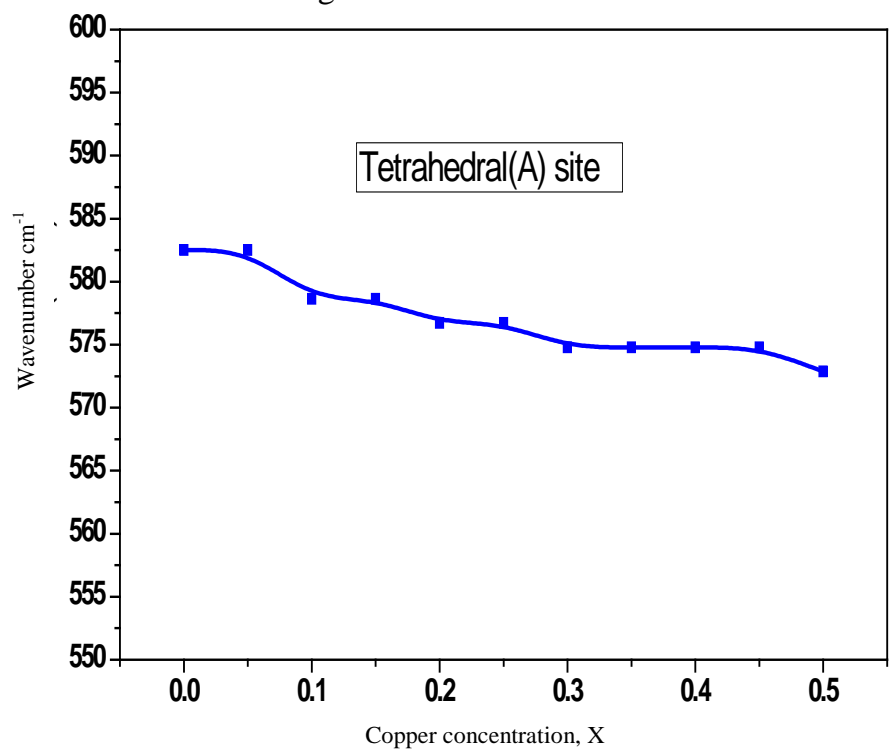

Figure 3. Variation of wave numbers at $\mathrm{A}$ and $\mathrm{B}$ sites with copper concentration.

$$
\mathrm{K}=4 \pi^{2} v^{2} \mathrm{C}^{2} \mathrm{~m}
$$

Where c is the speed of light, $\vartheta$ is the band wave number in $\mathrm{cm}^{-1}$ and $\mathrm{m}$ is the reduced mass for $\mathrm{Fe}^{3+}$ ions and $\mathrm{O}^{2-}$ ions $\left(2.061 \times 10^{-23} \mathrm{~g}\right)$. The values of force constant for tetrahedral $\left(\mathrm{K}_{\mathrm{T}}\right)$ and octahedral $\left(\mathrm{K}_{\mathrm{O}}\right)$ sites are listed in Table 2. The variation in force constant with copper concentration(x) for all samples at tetrahedral and octahedral sites is shown in Figure 4 and Figure 5 respectively. From the figure, it is observed that both $\mathrm{K}_{\mathrm{T}}$ and $\mathrm{K}_{\mathrm{O}}$ decrease with increase in copper concentration.

Table 2. Calculated force constants of tetrahedral and octahedral sites

\begin{tabular}{ccc}
\hline $\mathrm{x}$ & $\mathrm{K}_{\mathrm{T}}, \mathrm{x} 10^{5}$ dyne $\mathrm{cm}^{-1}$ & $\mathrm{~K}_{\mathrm{O}}, \mathrm{x} 10^{5}$ dyne $\mathrm{cm}^{-1}$ \\
\hline 0.00 & 2.49 & 1.18 \\
0.05 & 2.49 & 1.08 \\
0.10 & 2.45 & 1.07 \\
0.15 & 2.45 & 0.94 \\
0.20 & 2.44 & 1.15 \\
0.25 & 2.44 & 1.03 \\
0.30 & 2.42 & 0.99 \\
0.35 & 2.42 & 1.07 \\
0.40 & 2.42 & 1.11 \\
0.45 & 2.42 & - \\
0.45 & 2.40 & 1.00 \\
\hline
\end{tabular}




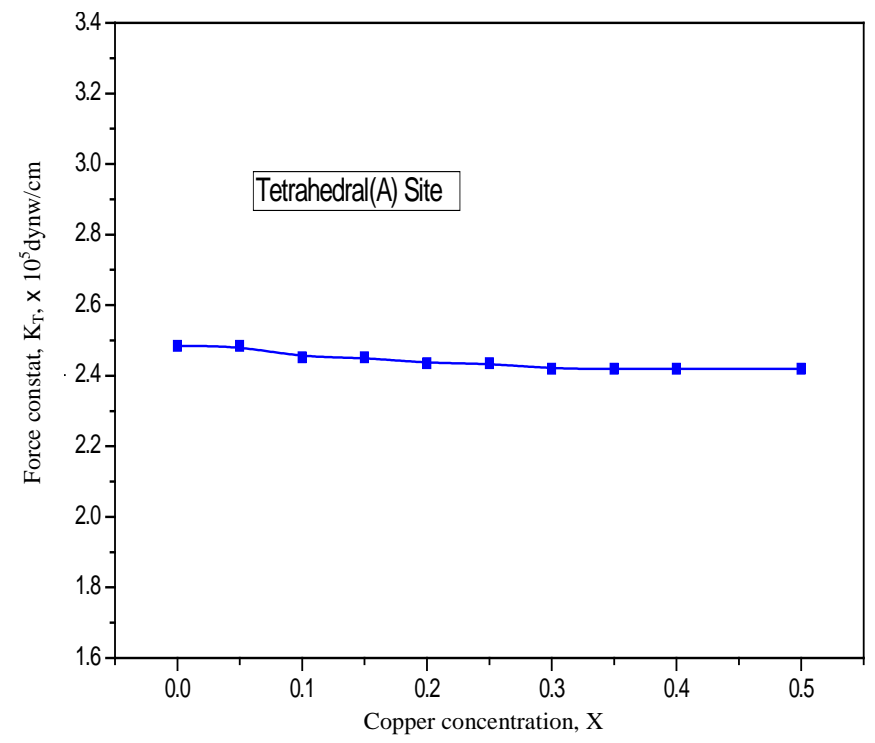

Figure 4. Variation in force constants with increasing copper concentration (x) at tetrahedral site

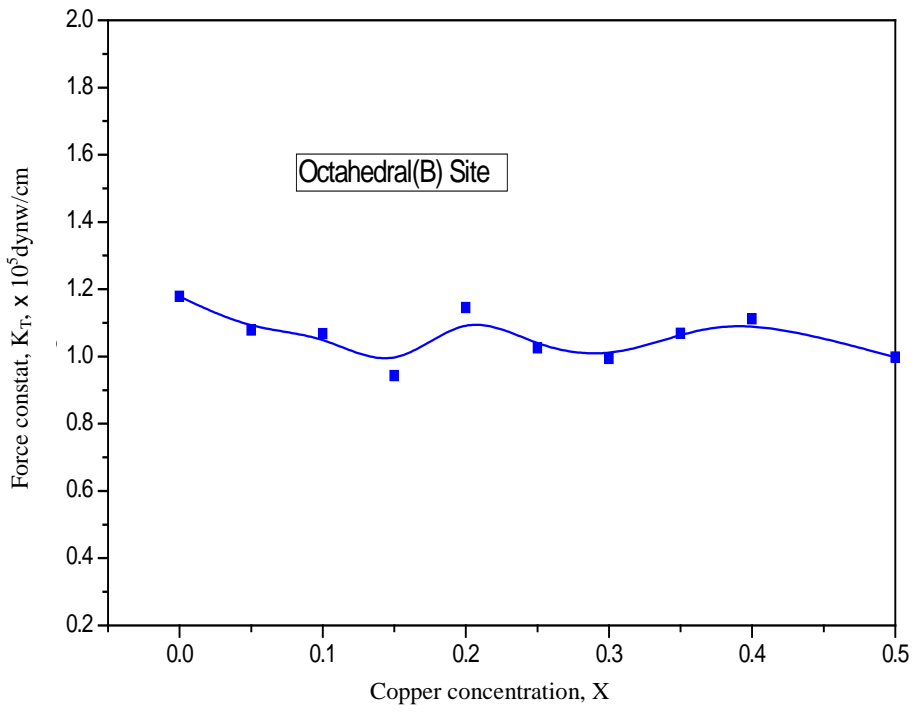

Figure 5. Variation in force constants with increasing copper concentration (x) at octahedral site

The change in the band position is due to the change in the $\mathrm{Fe}^{3+}-\mathrm{O}^{2-}$ inter nuclear distances for the tetrahedral and octahedral sites respectively ${ }^{13,14}$. Normally, it is expected that an increase in band length should lead to a decrease in force constant. If the radius of the impurity ion is larger than the displaced ion then the bond length increases, lowering the force constant for either site or a reduction in the repulsive forces between the ions leading to a lower electrostatic energy implying lower wave number. Reverse will hold if a smaller impurity ion replaces a metal ion of the regular lattice. A decrease in wave number and force constant is expected with copper substitution because of its larger ionic radius ${ }^{14}(0.72 \AA)$ than the displaced $\mathrm{Ni}^{2+}$ ion $(0.69 \AA)$. 
The observed change in wave numbers $\left(\vartheta_{1} \& \vartheta_{2}\right)$ and decrease in force constants $\left(\mathrm{K}_{\mathrm{T}} \&\right.$ $\mathrm{K}_{\mathrm{O}}$ ) which are composition depend at both the sites indicate the occupancy of copper ions at both A and B sites. The observed changes in intensities (Table 1) at both A and B sites also support the occupancy of copper ions at both A and B sites. But more change in these parameters at octahedral site than that at tetrahedral site suggests that occupancy of copper ions at $\mathrm{B}$ site is more than that at $\mathrm{A}$ site.

\section{Conclusion}

The room temperature infrared spectra of copper substituted Nickel-Zinc ferrite confirm the cubic spinel ferrite. All the doping samples clearly show the ferrite formation in the range from $300 \mathrm{~cm}^{-1}$ to $1000 \mathrm{~cm}^{-1}$. The change in the band position is due to the change in the $\mathrm{Fe}^{3+}-\mathrm{O}^{2-}$ inter nuclear distances for the tetrahedral and octahedral sites respectively. If the radius of the impurity ion is larger than the displaced ion then the bond length increases, lowering the force constant for either site or a reduction in the repulsive forces between the ions leading to a lower electrostatic energy implying lower wave number. Reverse will hold if a smaller impurity ion replaces a metal ion of the regular lattice. The frequency shift observed are reasonable due to replacement $\mathrm{Ni}^{2+}$ with $\mathrm{Cu}^{2+}$ ions having larger ionic radius which effects $\mathrm{Fe}^{3+}-\mathrm{O}^{2-}$. A decrease in wave number and force constant is expected with copper substitution because of its larger ionic radius $(0.72 \AA)$ than the displaced $\mathrm{Ni}^{2+}$ ion $(0.69 \AA)$.

\section{References}

1. R.P.Pant, Manju Arora, Balwinder Kaur, Vinod Kumar and Ashok Kumur $J$ Magnetism Magnetic Mater., 2010, 322, 3688-3691;

DOI:10.1016/j.jmmm.2010.07.026

2. Waldron R D, Phys Rev., 1955, B99, 1727.

3. West A R, Solid State Chemistry and its Applications; John Wiley \& Sons: London 1984.

4. $\quad$ Rao C N, Chem Appl IR Spec., Academic Press, 1963, 356.

5. $\quad$ Erum Pervaiz and Gul I H, J Magnetism Magnetic Materials, 2012, 324(22), 36953703; DOI:10.1016/j.jmmm.2012.05.050

6. Josyulu O S and Sobhanadri J, Phys Stat Sol., (a) 1981, 65(2), 479483; DOI:10.1002/pssa.2210650209

7. Hsiang H I, Chih-Cheng Chen and Yue Tsai W, Appl Surface Sci., 2005, 245(1-4), 252-259; DOI:10.1016/j.apsusc.2004.10.048

8. Binu P Jacob, Smitha Thankachan, Sheena Xavier and Mohammed E M, Phys Scripta., 2011, 84, 045702; DOI:10.1088/0031-8949/84/04/045702

9. Mariya J Hernandez- Moreno, Maria J Ulibarri, J L Rendon and Carlos J Serna, Phys Chem Min., 1985, 12. 34; DOI: 10.1007/BF00348744

10. Hair M L, J Non-Cryst Solids, 1975, 19, 299-309; DOI:10.1016/00223093(75)90095-2

11. Balavijayalakshmi J, Suriyanarayanan N and Jayaprakash R, J Mag Magn Mater., 2014, 362, 135-140; DOI:10.1016/j.jmmm.2014.03.005

12. Ahmed M A, Mansour S F and El-Dek S I. Physica B: Condensed Matter., 2008, 403(1), 224-230; DOI:10.1016/j.physb.2007.08.216

13. Shannon R D, Acta Cryst., 1976, A32, 751-767,

14. Verwey F J W and De Boer J H, Rec Trav Chem Pays Bas., 1936, 55, 531. 